\title{
Study on the Transmission Characteristics of Polarized Laser in the Complicated Dust Environment on Mars
}

\author{
Yu-Feng Yang $\mathbb{D},^{1,2}$ Jian Ke $\mathbb{D}^{1},{ }^{1}$ An-Li Han $\mathbb{D}^{1},{ }^{1}$ and Rui-Na Xu $\mathbb{D}^{1}$ \\ ${ }^{1}$ School of Automation \& Information Engineering, Xi'an University of Technology, Xi'an 710048, China \\ ${ }^{2}$ Shaanxi Civil-Military Collaboration Key Laboratory of Intelligence Coordination Networks, Xi'an University of Technology, \\ Xi'an 710048, China \\ Correspondence should be addressed to Jian Ke; 473432166@qq.com
}

Received 2 November 2021; Accepted 12 January 2022; Published 4 February 2022

Academic Editor: Heng Liu

Copyright (c) $2022 \mathrm{Yu}$-Feng Yang et al. This is an open access article distributed under the Creative Commons Attribution License, which permits unrestricted use, distribution, and reproduction in any medium, provided the original work is properly cited.

\begin{abstract}
In this paper, for the dust environment in complicated atmospheric on Mars, the Martian atmosphere is vertically stratified based on the vertical distribution characteristic of Martian dust particles. Combining the number concentration and particle size distribution of each layer of dust, the transmission characteristics of polarized laser in the Martian dust are studied by using the polarized Monte Carlo method. The results show that among the six selected wavelengths, the transmittance of the wavelength of $7.46 \mu \mathrm{m}$ in Martian dust is the largest, while the laser intensity attenuation of the wavelength of $0.49 \mu \mathrm{m}$ in Martian dust is the largest. The horizontally polarized laser of the wavelength of $0.55 \mu \mathrm{m}$ (effective variance $b=0.1 \mu \mathrm{m}$ ) is taken as an example; the depolarization degree of the polarized laser under stratified conditions increases from the minimum value of $3.5440157 e-4$ at $0 \mathrm{~km}$ to the maximum value of 0.0092563 at $24 \mathrm{~km}$, with an increase of 0.00890189843 ; while the depolarization degree under nonstratified conditions increases from the minimum value of $1.2678582 e-4$ at $0 \mathrm{~km}$ to the maximum value of 0.0045636 at $24 \mathrm{~km}$, with an increase of only 0.004436842 , the degree of depolarization under the condition of stratified is greater than that of nonstratified. On the whole, for different effective variance $b$, the variation trend of the polarization parameter with height for different polarized laser is basically identical.
\end{abstract}

\section{Introduction}

As the second-closest terrestrial planet to the Earth, Mars has always been the focus of deep-space exploration in the world. Mars has a complicated atmosphere and lacks water and vegetation on its surface. Almost every Martian year (equivalent to 687 days on Earth), there will be an extraordinary dust storm sweeping the globe on Mars, with a high speed of $180 \mathrm{~m} / \mathrm{s}$ and a long duration. The absorption and scattering effect of suspended dust particles in the Martian dust storm will cause laser attenuation, which will seriously affect the stability and reliability of wireless optical communication on Mars. In the near-surface atmosphere on Mars, the concentration and particle size of dust particles are unevenly distributed in vertical height, so it is necessary to perform stratification when calculating the transmission characteristics of laser. In this research field,
Wu et al. [1] in 2004 used the four-flux method and Monte Carlo method to study the multiple scattering and transmission attenuation characteristics of laser in a stratified dust atmosphere, which provided a theoretical basis for the numerical calculation of laser transmission in a dust environment. Compared with the traditional laser, the polarized laser is widely used in the field of laser communication and detection due to its unique characteristics [2, 3]. In terms of polarized laser research, Plass and Kattawar and Plass et al. [4, 5] from 1972 to 1973 used the Monte Carlo method to calculate and analyze the variation in polarization degree and polarization direction after multiple scattering of polarized laser in the clouds, which laid a foundation for the later detection of aerosol parameters in the atmosphere. In 2012, Yang [6] studied the depolarization effect of polarized laser in atmospheric transmission, which furnished a basis for the application of 
circular polarization coding in communication systems. In 2015, Fernández et al. [7] used polarized Monte Carlo to analyze the full polarization characteristic of the Rayleigh and Compton effects in multiple scattering. In 2019, Ma et al. [8] studied the transmission characteristics of the signal laser in free-space optical communication systems, which provided theoretical support for obtaining higher mixing efficiency by using a polarization control algorithm. In 2020, Yang et al. [9] used the polarized Monte Carlo method to simulate the multiple scattering of polarized laser in the Martian atmosphere, which offered theoretical support for analyzing the effect of wavelength, particle size distribution, wind speed, height, and particle number concentration on the transmission characteristics of the polarized laser. In 2021, Alemanno et al. [10] used spectral polarization technology to measure the optical constant of Mars simulated matter, which supplied a method for the planetary science community to derive the optical constant of mineral and material (natural or synthetic).

Based on the Martian atmospheric environment, this paper uses the polarized Monte Carlo method to stratify the atmosphere of $0-24 \mathrm{~km}$ on Mars and calculates the transmission characteristics of polarized laser at the vertical height. Then the transmittance and depolarization degree of polarized laser transmitted in the Martian dust environment are analyzed to provide theoretical support for the realization of reliable and stable wireless optical communication on Mars in the future.

\section{Laser Transmission Theory}

According to the complex refractive index of Martian dust particles [11,12], as shown in Table 1, Mie theory [13] is used to calculate the extinction efficiency factor $Q_{\text {ext }}$, scattering efficiency factor $Q_{\text {sca }}$, and absorption efficiency factor $Q_{a b s}$ of Martian dust particles as follows:

$$
\begin{aligned}
& Q_{\mathrm{sca}}=\frac{2}{x^{2}} \sum_{n=1}^{\infty}(2 n+1)\left(\left|a_{n}\right|^{2}+\left|b_{n}\right|^{2}\right), \\
& Q_{\mathrm{ext}}=\frac{2}{x^{2}} \sum_{n=1}^{\infty}(2 n+1)\left\{\left(a_{n}+b_{n}\right)\right\}, \\
& Q_{\mathrm{abs}}=Q_{\mathrm{ext}}-Q_{\mathrm{sca}} .
\end{aligned}
$$

Here, $a_{n}$ and $b_{n}$ represent the scattering coefficient, and the spectral scattering characteristic of Martian dust particles can be calculated by equation (1).

Based on the spectral scattering characteristic of Martian dust particles, combined with the laser transmission theory, the multiple scattering of polarized laser in Martian dust environment is imitated by the polarized Monte Carlo simulation method [14]. The specific steps are as follows [9]:

(1) Set the initial state of the photon, including position coordinate, movement direction, and so on.
(2) Movement of the photon. The photon moves from a point $(x, y, z)$ to the next collision point $\left(x^{\prime}, y^{\prime}, z^{\prime}\right)$. The relationship between the two points is as follows:

$$
\left\{\begin{array}{l}
x=x^{\prime}+\mu_{x} L, \\
y=y^{\prime}+\mu_{y} L, \\
z=z^{\prime}+\mu_{z} L .
\end{array}\right.
$$

Here, $\left(\mu_{x}, \mu_{y}, \mu_{z}\right)$ is the cosine of the direction angle; $L$ is the moving step length, $L=-\ln (\xi) / \mu_{t} ; \xi$ is a random number evenly distributed between 0 and 1 ; and $\mu_{t}$ is the average attenuation coefficient of the particles.

(3) Selection of scattering direction. Suppose the Stokes vector of the incident wave is $S_{0}=\left[I_{0}, Q_{0}, U_{0}, V_{0}\right]^{\prime}$, then its phase function is as follows:

$$
\begin{aligned}
p(\alpha, \beta)= & m_{11}(\alpha) I_{0}+m_{12}(\alpha) \\
& {\left[Q_{0} \cos (2 \beta)+U_{0} \sin (2 \beta)\right] . }
\end{aligned}
$$

Here, $m_{11}(\alpha)$ and $m_{12} \underline{(\alpha)}$ are the two elements of the scattering matrix $\bar{M}(\alpha), \alpha$ is the scattering angle, and $\beta$ is the rotation angle between the incident meridian surface and the scattering surface. Then use the "acceptance-rejection sampling" method to sample the random variable $\alpha_{r}$, $\beta_{r}$, and $p_{r}$ in equation (2), and the corresponding interval is $(0, \pi),(0,2 \pi)$, and $(0,1)$. If $P_{r} \leq P\left(\alpha_{r}, \beta_{r}\right)$, then the selection is successful, and go to the next step; otherwise, select again.

(4) Photon scattering tracking and polarization processing. After the scattering angle and azimuth are successfully selected, the Stokes parameter will be converted in three steps:

(i) From the incident meridian surface to the scattering surface, the incident Stokes parameter $S$ is multiplied by the rotation matrix $\overline{\bar{L}}(\beta)$ to obtain the rotated Stokes parameter $\bar{S}_{1}$; the calculation of $\overline{\bar{L}}(\beta)$ is as follows:

$$
\overline{\bar{L}}(\beta)=\left[\begin{array}{l}
1 \\
0 \\
0 \\
0
\end{array}\right] .
$$

(ii) Transformation on the scattering surface. The Stokes parameter is transferred from one point to another on the scattering surface, and the conversion matrix is Mueller matrix $\bar{M}(\alpha)$. At the same time, the conversion of the direction angle cosine is performed, and the new direction cosine is $\left(\widehat{u}_{x}, \widehat{u}_{y}, \widehat{u}_{z}\right)$.

(iii) Return from the scattering surface to the new meridian surface to reach the final goal. The 
TABLE 1: The complex refractive index of Martian dust particles at typical wavelengths $[11,12]$.

\begin{tabular}{lcccccc}
\hline$\lambda / \mu \mathrm{m}$ & 0.49 & 0.55 & 0.66 & 0.86 & 7.46 & 10.6 \\
\hline Complex refractive index & $1.52+0.01 i$ & $1.52+0.007 i$ & $1.52+0.0025 i$ & $1.52+0.0065 i$ & $1.16+0.06 i$ & $2.5+0.16 i$ \\
\hline
\end{tabular}

rotation matrix at this time is $\overline{\bar{L}}(-\gamma)$, which is calculated as follows:

$$
\cos \gamma=\frac{-\mu_{z}+\widehat{\mu}_{z}}{ \pm \sqrt{\left(1-\cos \alpha^{2}\right)\left(1-\widehat{\mu}_{z}^{2}\right)}} .
$$

If $\beta \in(\pi, 2 \pi)$, equation (5) takes the plus sign. If $\beta \in(0, \pi)$, equation (5) takes the minus sign. The final Stokes parameter is shown in the following equation [15]:

$$
\bar{S}_{0}=\overline{\bar{L}}(-\gamma) \overline{\bar{M}}(\alpha) \overline{\bar{L}}(\beta) \bar{S} .
$$

Here, $\bar{S}$ represents the Stokes parameter before scattering.

(5) Termination condition and final state. The tracking of the photon is terminated when the photon escapes the interface or the weight $W$ is less than the set value. The final state of the total polarized laser is as follows:

$$
\bar{S}=\frac{1}{N} \sum_{n=1}^{N} \bar{S}_{n}
$$

Here, $N$ is the number of photons. The quantitative value describing the degree of polarization of polarized laser is expressed by the degree of polarization $P$ as follows [16]:

$$
P=\frac{\sqrt{Q^{2}+U^{2}+V^{2}}}{I} .
$$

Here, $I$ represents the total laser intensity, $Q$ represents the difference value of laser intensity in $x$ and $y$ directions, $U$ represents the completely linearly polarized laser component with an angle of $\pm 45^{\circ}$ to the scattering plane, $V$ represents the right- or left-hand polarized state component of the circularly polarized laser, and $P$ represents the proportion of the laser intensity of completely linearly polarized laser in the total laser intensity. Use the degree of depolarization to describe the variation in the degree of polarization of linearly polarized laser, as follows:

$$
\text { Dep }=\frac{I_{\perp}}{I_{p}} \times 100 \% .
$$

Here, $I_{p}=(I+Q) / 2$ represents the laser intensity in the horizontal component and $I_{\perp}=(I-Q) / 2$ represents the laser intensity in the vertical component.

Because the concentration and particle size of dust particles in the near-surface atmosphere on Mars are unevenly distributed in the vertical height, it is necessary to stratify the lower atmosphere of Mars when calculating the transmission characteristics of the laser. For the polarized Monte Carlo processing of stratified Martian dust atmosphere, it is necessary to consider not only the reflection and transmission within each stratified surface but also the reflection and transmission relationship between adjacent layers. In the case of multiple scattering, it is assumed that photon moves from i layer to $i$ th +1 layer. At this time, it needs to be handled according to the following steps [1]: if the photon is transmitted to the $i$ th +1 layer, it is moved first to the boundary of the $i$ th +1 layer along the scattering direction; the transmission probability and weight function are calculated; and its transmission direction is decided, and then the photon travels the remaining distance along the new scattering direction. If the photon is not transmitted out and remains in the $i$ th layer, the parameter of this layer is used to continually track the position, scattering direction and transmission path of the next scattering; If the photon is transmitted to another layer, the above steps are repeated until the photon is absorbed in one layer or transmitted out of the dust.

2.1. Vertical Distribution of Martian Dust Particles. According to the launched Mars probes Viking 1 and Viking 2, the Martian atmosphere below $300 \mathrm{~km}$ is divided into troposphere, mesosphere, thermosphere, and exosphere from bottom to top. Because the concentration of Martian dust particles in the Martian atmosphere above $24 \mathrm{~km}$ is very small and almost negligible, this paper mainly studies that the Martian lower atmospheric communication environment under the height of $24 \mathrm{~km}$ has an influence on the transmission characteristics of the laser.

The topography of Mars has different aerodynamics in different regions. Dust storms of different sizes will be generated when the wind speed is different. In 2001, the Mars Global Surveyor probe revealed the entire process of the global dust storm on Mars. The results showed that the dust storm had already bypassed Mars in just 15 days, and its expansion speed was $16 \mathrm{~m} / \mathrm{s}$. Chassefière et al. [12] studied the influence of height variation on the distribution of dust particles in the Martian atmosphere and found that different size distribution, effective radius, and variance have different particle numbers and concentrations at different heights. The distribution of dust particles in three different models is measured, and the relationship of its distribution with height, effective radius, and variance is shown in Table 2 $[17,18]$.

\subsection{Attenuation Characteristic in the Vertical Direction of the} Martian Atmosphere. If the number concentration of Martian dust particles is taken as a constant, the calculated transmission attenuation error is too large because the effective radius and particle number concentration of Martian dust particles vary with height [19]. When there is no dust storm, the lower atmosphere of Mars is divided into 24 layers 
TABLE 2: The vertical distribution of the number concentration and effective radius of Martian dust particles corresponding to the three models $[17,18]$.

\begin{tabular}{lcccccc}
\hline \multirow{2}{*}{ Height $/ \mathrm{km}$} & \multicolumn{2}{c}{ Case $1: b=0.10$} & \multicolumn{2}{c}{ Case $2: b=0.25$} & \multicolumn{2}{c}{ Case 3: $b=0.40$} \\
& $n / \mathrm{cm}^{3}$ & $r_{\mathrm{eff}} / \mu \mathrm{m}$ & $n / \mathrm{cm}^{3}$ & $r_{\mathrm{eff}} / \mu \mathrm{m}$ & $n / \mathrm{cm}^{3}$ & $r_{\mathrm{eff}} / \mu \mathrm{m}$ \\
\hline 0 & 1.31 & 1.76 & 2.26 & 1.90 & 5.56 & 2.05 \\
1 & 1.18 & 1.75 & 2.06 & 1.88 & 5.09 & 2.03 \\
2 & 1.07 & 1.74 & 1.88 & 1.87 & 4.67 & 2.01 \\
3 & 0.97 & 1.73 & 1.71 & 1.85 & 4.28 & 1.99 \\
4 & 0.87 & 1.72 & 1.56 & 1.84 & 3.93 & 1.97 \\
5 & 0.79 & 1.71 & 1.43 & 1.82 & 3.62 & 1.94 \\
6 & 0.72 & 1.70 & 1.30 & 1.80 & 3.34 & 1.91 \\
7 & 0.65 & 1.68 & 1.19 & 1.78 & 3.08 & 1.88 \\
8 & 0.59 & 1.67 & 1.09 & 1.75 & 2.86 & 1.85 \\
9 & 0.53 & 1.65 & 1.00 & 1.73 & 2.65 & 1.81 \\
10 & 0.48 & 1.64 & 0.92 & 1.70 & 2.47 & 1.78 \\
11 & 0.43 & 1.62 & 0.85 & 1.67 & 2.31 & 1.74 \\
12 & 0.39 & 1.60 & 0.78 & 1.64 & 2.16 & 1.69 \\
13 & 0.36 & 1.58 & 0.72 & 1.61 & 2.04 & 1.65 \\
14 & 0.33 & 1.55 & 0.68 & 1.57 & 1.94 & 1.60 \\
15 & 0.30 & 1.53 & 0.64 & 1.54 & 1.86 & 1.55 \\
16 & 0.27 & 1.50 & 0.60 & 1.49 & 1.79 & 1.50 \\
17 & 0.25 & 1.47 & 0.56 & 1.45 & 1.72 & 1.44 \\
18 & 0.23 & 1.44 & 0.52 & 1.41 & 1.65 & 1.38 \\
19 & 0.20 & 1.41 & 0.48 & 1.36 & 1.56 & 1.33 \\
20 & 0.18 & 1.38 & 0.44 & 1.32 & 1.47 & 1.27 \\
21 & 0.16 & 1.34 & 0.40 & 1.26 & 1.40 & 1.20 \\
22 & 0.14 & 1.29 & 0.38 & 1.20 & 1.32 & 1.14 \\
23 & 0.13 & 1.25 & 0.35 & 1.15 & 1.25 & 1.08 \\
24 & 0.12 & 1.20 & 0.33 & 1.09 & 1.20 & 1.02 \\
\hline & & & & & &
\end{tabular}

according to Table 1 . The 1st layer has the lowest height and the biggest particle concentration; the particle number concentration is $1.31 \mathrm{n} / \mathrm{cm}^{3}, 2.26 \mathrm{n} / \mathrm{cm}^{3}$, and $5.56 \mathrm{n} / \mathrm{cm}^{3}$ (the effective variance is $b=0.10 \mu \mathrm{m}, b=0.25 \mu \mathrm{m}$, and $b=0.40 \mu \mathrm{m}$, respectively); the 24th layer has the highest height, but its particle number concentration is the lowest; the particle number concentration is $0.12 \mathrm{n} / \mathrm{cm}^{3}, 0.33 \mathrm{n} / \mathrm{cm}^{3}$, and $1.20 \mathrm{n} / \mathrm{cm}^{3}$ (the effective variance is $b=0.10 \mu \mathrm{m}$, $b=0.25 \mu \mathrm{m}$, and $b=0.40 \mu \mathrm{m}$, respectively). The polarized Monte Carlo method is used to calculate and analyze the laser transmission under two conditions (stratified and nonstratified). The particle number concentration is fixed to $0.39 \mathrm{n} / \mathrm{cm}^{3} 0.78 \mathrm{n} / \mathrm{cm}^{3}$, and $2.16 \mathrm{n} / \mathrm{cm}^{3}$ (the effective variance is $b=0.10 \mu \mathrm{m}, b=0.25 \mu \mathrm{m}$, and $b=0.40 \mu \mathrm{m}$, respectively) under the condition of nonstratified, and the effective radius is taken, respectively, as $1.6 \mu \mathrm{m}, 1.64 \mu \mathrm{m}$, and $1.69 \mu \mathrm{m}$. The calculated results are shown in Table 2, which is the transmission transmittance corresponding to several typical wavelengths under the condition of stratified and nonstratified.

It can be seen from Table 3 that when the wavelength is $7.46 \mu \mathrm{m}$, the calculated transmittance is larger than that of other wavelengths because the real part of the complex refractive index of this wavelength is the smallest among several typical wavelengths, and its transmittance reaches the maximum value of $9.7939100 e-001$ when the effective variance $b$ is $0.1 \mu \mathrm{m}$. On the contrary, the real part of the complex refractive index of the wavelength of $10.6 \mu \mathrm{m}$ is larger than that of other wavelengths, so the calculated transmittance at this wavelength is the smallest, and its transmittance reaches the minimum value of $8.3379000 e-002$ when the effective variance $b$ is $0.4 \mu \mathrm{m}$. The transmittance of different wavelengths is an obvious difference, and the calculated result is also different between the condition of stratified and nonstratified, but the calculated result under the condition of stratified is more accurate than that of nonstratified, which is more in line with the actual dust environment on Mars.

\section{Transmission Characteristics of Different Polarized Laser in Martian Dust Environment}

Then the transmission characteristics of the horizontal and vertical components of different polarized laser are studied in the Martian dust. The distributed parameter is $r_{\text {eff }}=2.34 \mu \mathrm{m}$ and $v_{\text {eff }}=0.86 \mu \mathrm{m}$, and the particle number concentration is $1.8 \times 10^{9} / \mathrm{m}^{3}$ at the height of $18 \mathrm{~km}$. The calculation results are shown in Figures 1-5.

Figures 1(a) and 1(b), respectively, show the variation curves of the horizontal and vertical components with the transmission distance when horizontally polarized laser [1, $1,0,0]$ transmitted in the Martian dust. Figures 2(a) and 2(b), respectively, show that the variation curves of the horizontal and vertical components with the transmission distance when vertically polarized laser $[1,-1,0,0]$ transmitted in the Martian dust; it almost symmetrically coincides with the variation curves of horizontally polarized light $[1,1$, $0,0]$ in the Martian dust. As can be seen from Figure 2(b), with the increase of transmission distance, the laser intensity in the vertical component increases first and reaches a peak at about $1 \mathrm{~km}$ and then decreases continually. In summary, when horizontally and vertically polarized laser transmitted in the Martian dust, as the transmission distance increases, the vertical and horizontal laser intensity will increase first to a peak and then decrease. The intensity reduction in the horizontal component and the intensity increase in the vertical component at the wavelength of $7.46 \mu \mathrm{m}$ are significantly smaller than those of other wavelengths [9].

Figures 3(a) and 3(b), respectively, show the variation curves of the horizontal and vertical components with the transmission distance when completely linearly polarized laser $[1,0,1,0]$ (with an angle of $45^{\circ}$ to the scattering plane) transmitted in the Martian dust. Figures 4(a) and 4(b), respectively, show the variation curves of the horizontal and vertical components with the transmission distance when completely linearly polarized laser $[1,0,-1,0]$ (with an angle of $-45^{\circ}$ to the scattering plane.) transmitted in the Martian dust.

It can be seen from Figure 3 that both horizontal and vertical components of completely linearly polarized laser [1, $0,1,0]$ (with an angle of $45^{\circ}$ to the scattering plane) gradually decrease with the increase of the transmission distance. Among the six typical wavelengths, the laser intensity attenuation in the horizontal and vertical components at the wavelength of $7.46 \mu \mathrm{m}$ is the lowest. From $0.4 \mathrm{~km}$ to $4 \mathrm{~km}$, the laser intensity of the horizontal component drops from 0.1081 to 0.0576 , and its laser intensity attenuation is only 0.0505 . On the contrary, the laser intensity in the horizontal 
TABLE 3: Transmission transmittance of several typical wavelength (stratified and nonstratified).

\begin{tabular}{|c|c|c|c|c|}
\hline Wavelength $(\mu \mathrm{m})$ & $b(\mu \mathrm{m} ;$ variance $)$ & Complex refractive index & Transmissivity (stratified) & Transmissivity (nonstratified) \\
\hline \multirow{3}{*}{0.55} & 0.10 & \multirow{3}{*}{$1.52+0.007 i$} & $7.9103600 e-001$ & $8.4216980 e-001$ \\
\hline & 0.25 & & $6.0289500 e-001$ & $7.1565000 e-001$ \\
\hline & 0.40 & & $2.1583800 e-001$ & $3.8582656 e-001$ \\
\hline \multirow{3}{*}{0.49} & 0.10 & \multirow{3}{*}{$1.52+0.01 i$} & $7.7459200 e-001$ & $8.4949872 e-001$ \\
\hline & 0.25 & & $5.9623376 e-001$ & $7.0203491 e-001$ \\
\hline & 0.40 & & $2.3726077 e-001$ & $3.3753995 e-001$ \\
\hline \multirow{3}{*}{0.66} & 0.10 & \multirow{3}{*}{$1.52+0.0025 i$} & $7.5852800 e-001$ & $8.2223085 e-001$ \\
\hline & 0.25 & & $5.8008100 e-001$ & $6.8605071 e-001$ \\
\hline & 0.40 & & $2.3055895 e-001$ & $2.9494579 e-001$ \\
\hline \multirow{3}{*}{0.86} & 0.10 & \multirow{3}{*}{$1.52+0.0065 i$} & $7.7644400 e-001$ & $8.2275485 e-001$ \\
\hline & 0.25 & & $6.1219900 e-001$ & $6.8489371 e-001$ \\
\hline & 0.40 & & $2.3087800 e-001$ & $3.5274570 e-001$ \\
\hline \multirow{3}{*}{7.46} & 0.10 & \multirow{3}{*}{$1.16+0.06 i$} & $9.6841000 e-001$ & $9.7939100 e-001$ \\
\hline & 0.25 & & $9.3185400 e-001$ & $9.5545800 e-001$ \\
\hline & 0.40 & & $8.0279000 e-001$ & $8.7093000 e-001$ \\
\hline \multirow{3}{*}{10.6} & 0.10 & \multirow{3}{*}{$2.5+0.16 i$} & $7.7016000 e-001$ & $8.6791700 e-001$ \\
\hline & 0.25 & & $4.9239800 e-001$ & $7.1660600 e-001$ \\
\hline & 0.40 & & $8.3379000 e-002$ & $3.2057500 e-001$ \\
\hline
\end{tabular}

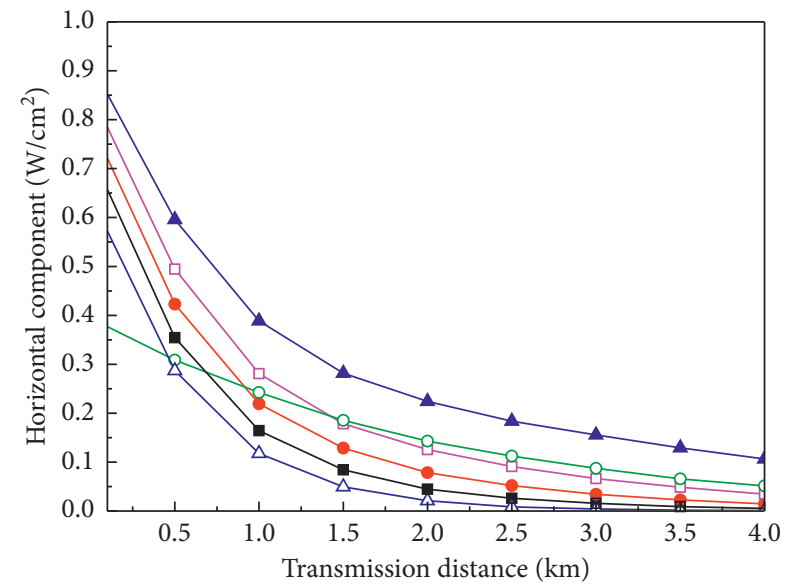

Wavelength $(\mu \mathrm{m})$

$$
\begin{array}{ll}
\rightarrow 0.49 & -\square 0.86 \\
\rightarrow 0.55 & -\circ 7.46 \\
\simeq 0.66 & -\square 10.6
\end{array}
$$

(a)

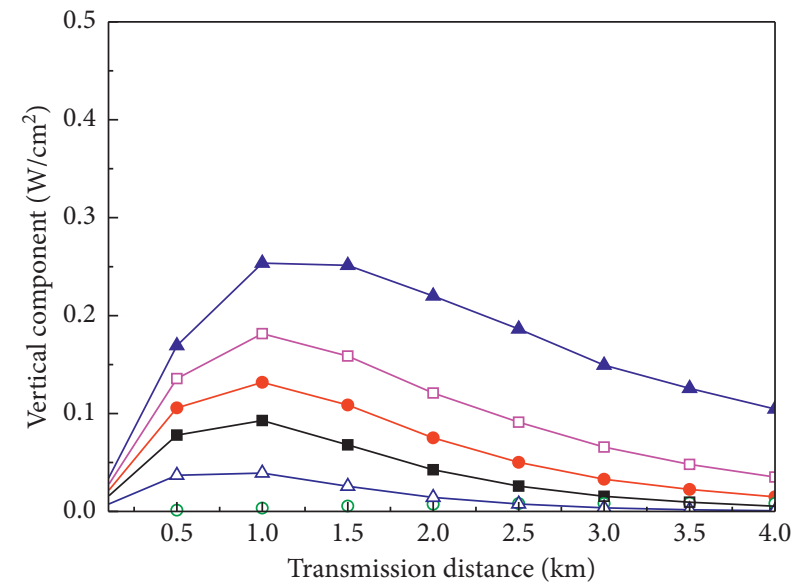

Wavelength $(\mu \mathrm{m})$

$$
\begin{array}{ll}
\rightarrow 0.49 & -\square 0.86 \\
\neg 0.55 & -\square 7.46 \\
\simeq 0.66 & -\square 10.6
\end{array}
$$

(b)

FiguRE 1: Horizontally polarized laser $[1,1,0,0]$ transmitted in the Martian dust: (a) horizontal component of horizontally polarized laser $[1,1,0,0]$ varying with the transmission distance and (b) vertical component of horizontally polarized laser $[1,1,0,0]$ varying with the transmission distance.

component drops from 0.33157 to 0.04651 , and the laser intensity attenuation of the wavelength of $0.49 \mu \mathrm{m}$ is 0.2851 , which also shows that polarized laser at this wavelength is most unsuitable for transmission in Martian dust environment. As can be seen from Figure 4, it is basically consistent with the variation trend of Figure 3 because completely linearly polarized laser $[1,0,1,0]$ (with an angle of $45^{\circ}$ to the scattering plane) is symmetrical with completely linearly polarized laser $[1,0,-1,0]$ (with an angle of $-45^{\circ}$ to the scattering plane), so their transmission characteristics are symmetrically coincident.
Figures 5(a) and 5(b), respectively, show the variation curves of the horizontal and vertical components with the transmission distance when circularly polarized laser $[1,0,0$, 1] (in a completely right-hand circularly polarized state) transmitted in the Martian dust. Figures 6(a) and 6(b), respectively, show the variation curves of the horizontal and vertical components with the transmission distance when circularly polarized laser $[1,0,0,-1]$ (in a completely lefthand circularly polarized state) transmitted in the Martian dust. Because the completely right-hand circularly polarized laser $[1,0,0,1]$ is symmetrical with the completely left-hand 


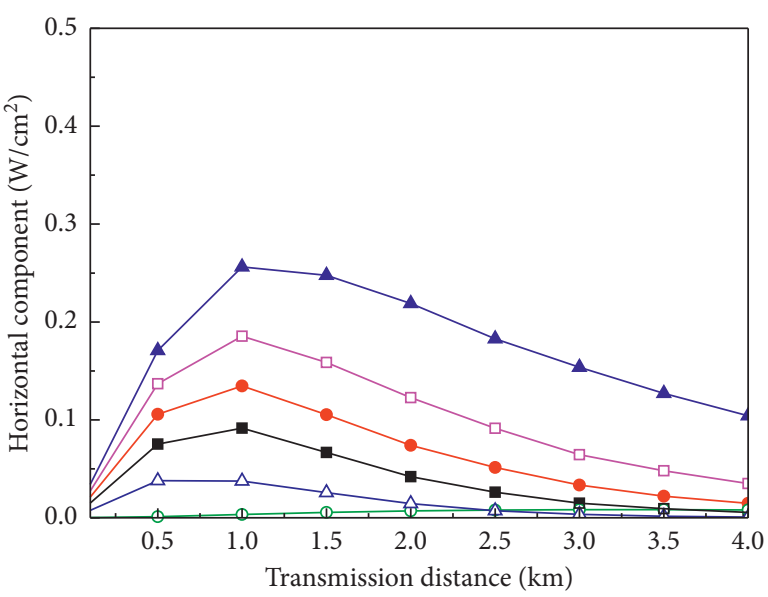

Wavelength $(\mu \mathrm{m})$

$$
\begin{array}{ll}
\rightarrow 0.49 & -\square 0.86 \\
\longrightarrow 0.55 & -\circ 7.46 \\
-0.66 & -\triangle 10.6
\end{array}
$$

(a)

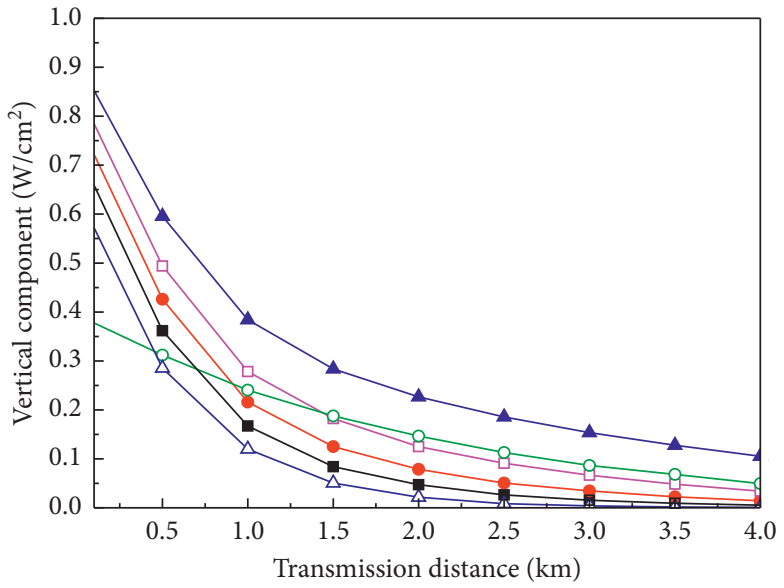

$$
\begin{array}{ll}
\text { Wavelength }(\mu \mathrm{m}) & \\
\rightarrow-0.49 & -\square 0.86 \\
\multimap 0.55 & -07.46 \\
\neg-0.66 & -\triangle 10.6
\end{array}
$$

(b)

FIGURE 2: Vertically polarized laser $[1,-1,0,0]$ transmitted in the Martian dust: (a) horizontal component of vertically polarized laser $[1,-1,0,0]$ varying with the transmission distance and (b) vertical component of vertically polarized laser $[1,-1,0,0]$ varying with the transmission distance.

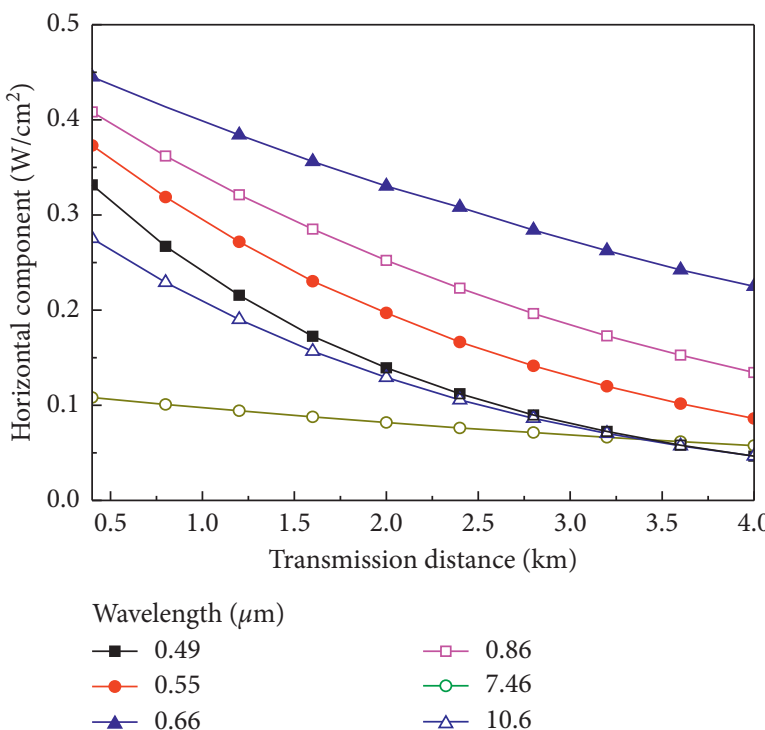

(a)

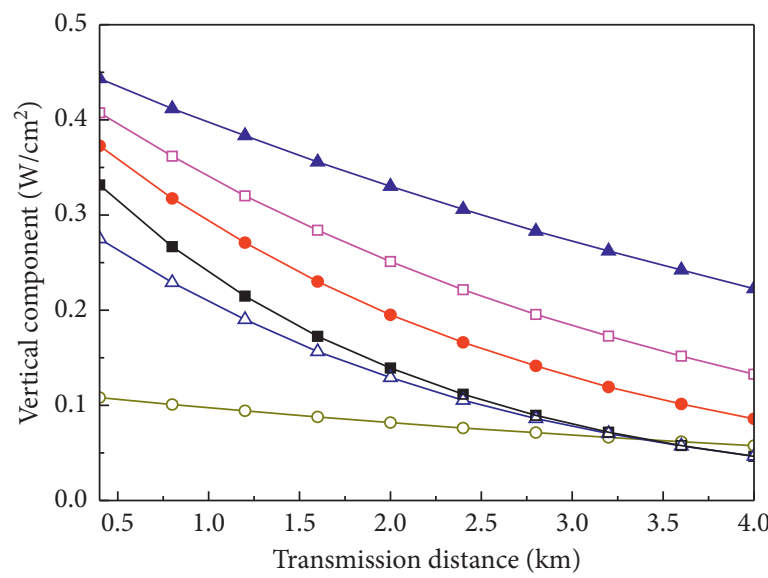

Wavelength $(\mu \mathrm{m})$

$\begin{array}{ll}\rightarrow 0.49 & -\square 0.86 \\ \multimap 0.55 & -\circ 7.46 \\ \leftarrow 0.66 & \square-10.6\end{array}$

(b)

FIgURE 3: Completely linearly polarized laser $[1,0,1,0]$ (with an angle of $45^{\circ}$ to the scattering plane) transmitted in the Martian dust: (a) horizontal component of completely linearly polarized laser $[1,0,1,0]$ varying with the transmission distance and (b) vertical component of completely linearly polarized laser $[1,0,1,0]$ varying with the transmission distance.

circularly polarized laser $[1,0,0,-1]$, their transmission characteristics are also symmetrically coincident.

Considering Figures 1-6, when horizontally polarized laser and vertically polarized laser transmitted in the Martian dust, the laser intensity variation in the horizontal and vertical components is different from that of other polarized laser; they will increase first to a peak and then decrease with the increase of transmission distance. But, when circularly polarized laser and completely linearly polarized laser transmitted in the Martian dust, their laser intensity in the horizontal and vertical components will gradually decrease with the increase of transmission distance. This is one of the reasons why horizontally and vertically polarized laser is often used to transmit in the Martian dust. In addition, among the selected wavelengths, no matter that polarized laser transmitted in the Martian dust, the laser intensity attenuation in the horizontal and vertical components of the wavelength of $7.46 \mu \mathrm{m}$ is the smallest. 


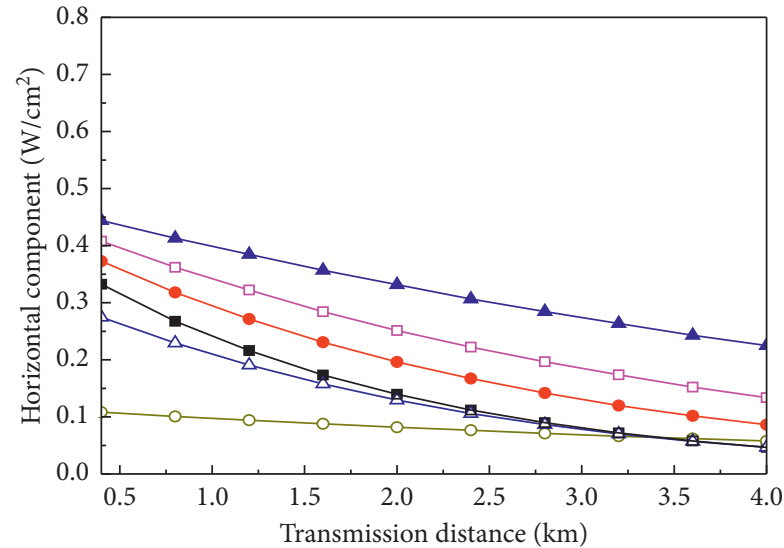

Wavelength $(\mu \mathrm{m})$

$\begin{array}{ll}\rightarrow 0.49 & -\square 0.86 \\ \rightarrow 0.55 & -\circ 7.46 \\ \triangle 0.66 & -\triangle 10.6\end{array}$

(a)

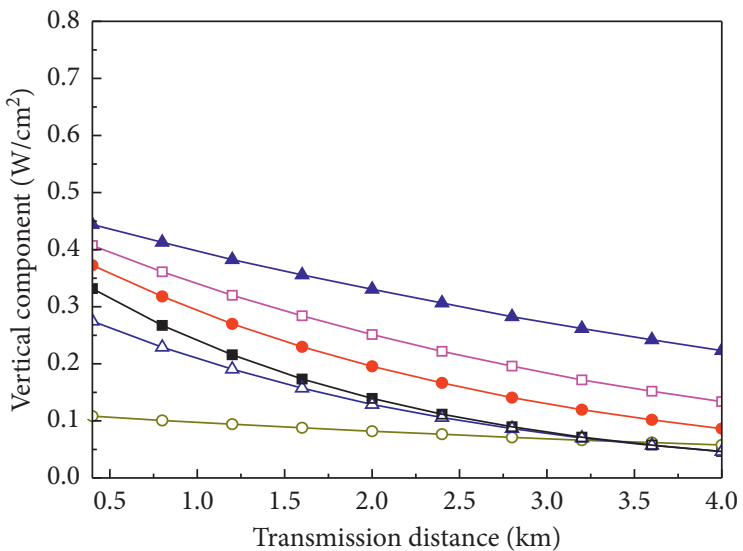

Wavelength $(\mu \mathrm{m})$

$\begin{array}{ll}\rightarrow 0.49 & -\square 0.86 \\ \rightarrow 0.55 & -\square 7.46 \\ \multimap 0.66 & -\square 10.6\end{array}$

(b)

Figure 4: Completely linearly polarized laser $[1,0,-1,0]$ (with an angle of $-45^{\circ}$ to the scattering plane) transmitted in the Martian dust: (a) horizontal component of completely linearly polarized laser $[1,0,-1,0]$ varying with the transmission distance and (b) vertical component of completely linearly polarized laser $[1,0,-1,0]$ varying with the transmission distance.

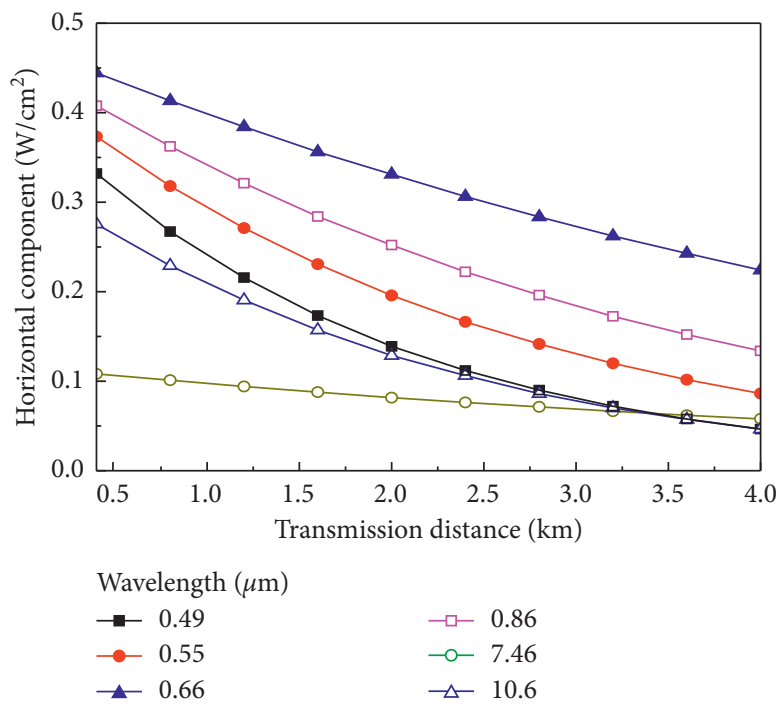

(a)

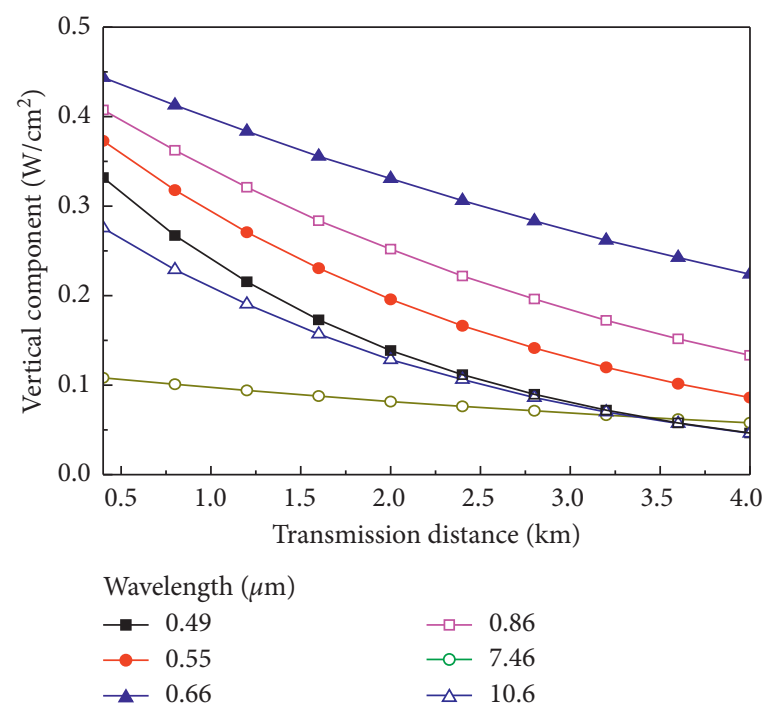

(b)

Figure 5: Circularly polarized laser $[1,0,0,1]$ (in a completely right-hand circularly polarized state) transmitted in. the Martian dust: (a) horizontal component of circularly polarized laser $[1,0,0,1]$ varying with the transmission distance and (b) vertical component of circularly polarized laser $[1,0,0,1]$ varying with the transmission distance.

\section{Transmission Characteristics of Polarized Laser in the Vertical Direction of the Martian Atmosphere}

Based on the content of Section 3, the transmission characteristics of polarized laser are studied in the vertical direction of Mars. Firstly, the horizontally polarized laser $[1,1,0,0]$ at the wavelength of $0.55 \mu \mathrm{m}$ is taken as an example; the value of different polarization parameters is calculated under the condition of stratified and nonstratified; and the calculation results are shown in Table 4. In the comparison table of polarization parameters.

It can be seen from the calculation results in Table 4 that the reflection parameter IR1 and degree of polarization $P 1$ under the condition of stratified are significantly less than those of nonstratified and the transmission parameter IT1 and degree of depolarization dep1 under the condition of stratified are greater than those of nonstratified. It shows that the depolarization 

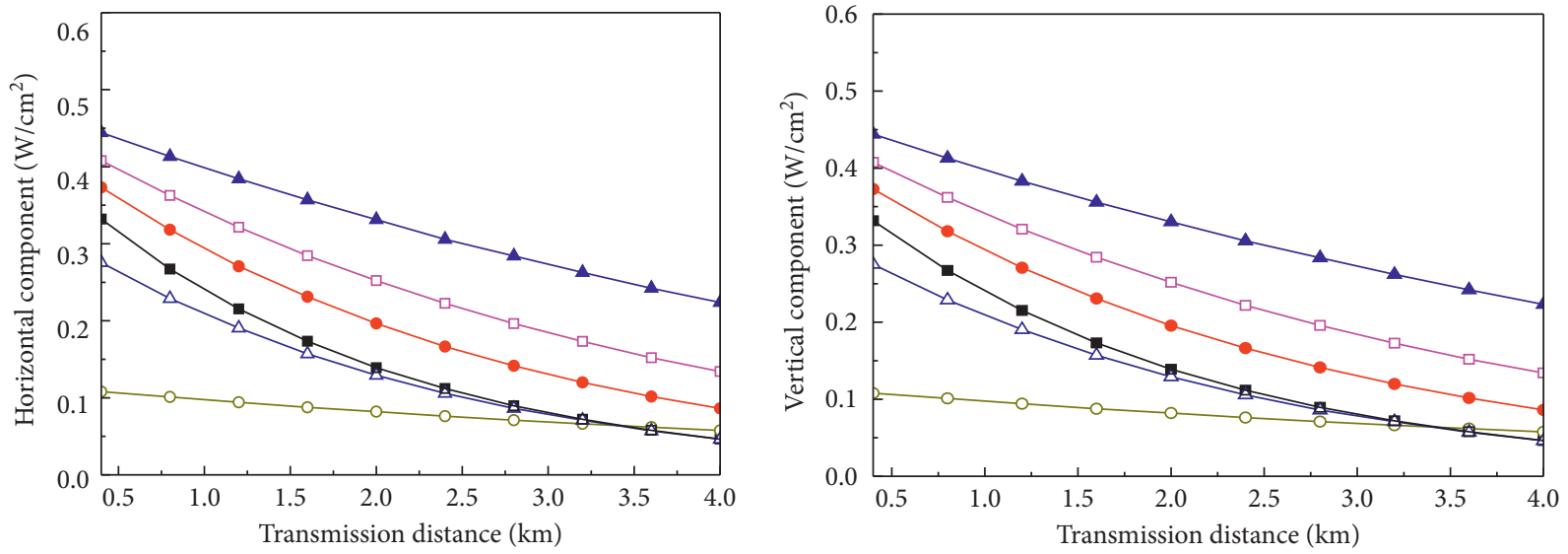

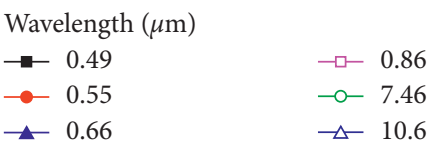

(a)

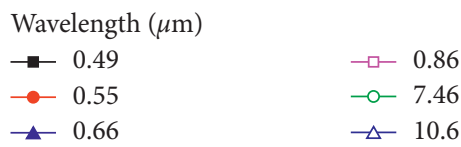

(b)

FIgURE 6: Circularly polarized laser [1, 0, 0, -1] (in a completely left-hand circularly polarized state) transmitted in the Martian dust: (a) horizontal component of circularly polarized laser $[1,0,0,-1]$ varying with the transmission distance and (b) vertical component of circularly polarized laser $[1,0,0,-1]$ varying with the transmission distance.

TABLE 4: Comparison table of polarization parameters (stratified and nonstratified).

\begin{tabular}{|c|c|c|c|c|c|c|c|c|}
\hline \multirow[t]{2}{*}{$b / \mu \mathrm{m}($ Variance $)$} & \multicolumn{4}{|c|}{ Stratified } & \multicolumn{4}{|c|}{ Nonstratified } \\
\hline & IR1 & IT1 & dep1 & $P 1$ & IR & IT & dep & $P$ \\
\hline 0.10 & 0.00181 & 0.99255 & 0.01011 & 0.97998 & 0.00308 & 0.77026 & 0.00491 & 0.99024 \\
\hline 0.25 & 0.00365 & 0.98462 & 0.02240 & 0.95619 & 0.00699 & 0.73117 & 0.01250 & 0.97523 \\
\hline 0.40 & 0.00923 & 0.95513 & 0.0627 & 0.88198 & 0.01603 & 0.61652 & 0.06925 & 0.86989 \\
\hline
\end{tabular}

IR represents the total laser intensity in the reflection parameter; $b$ represents effective variance; IT represents the total laser intensity in the transmission parameter; dep represents the degree of depolarization; and $P$ represents the degree of polarization.

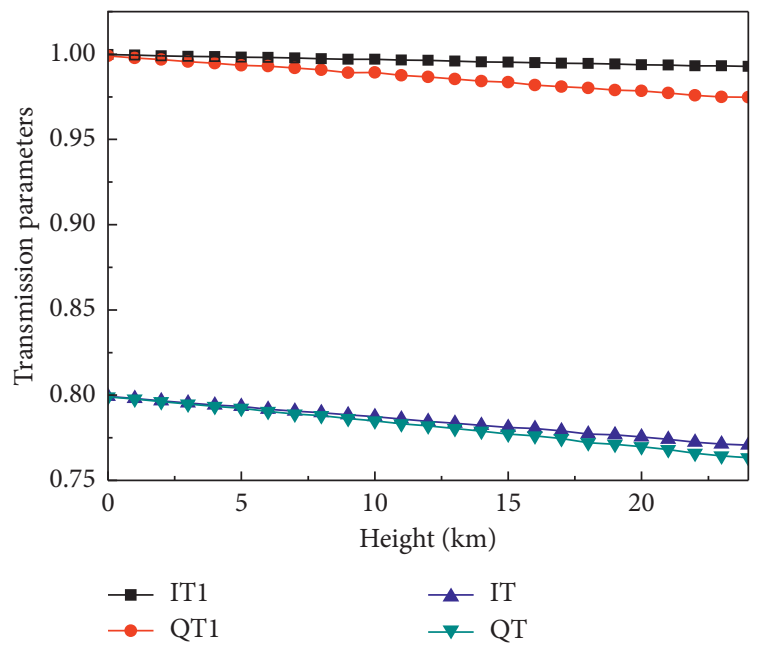

(a)

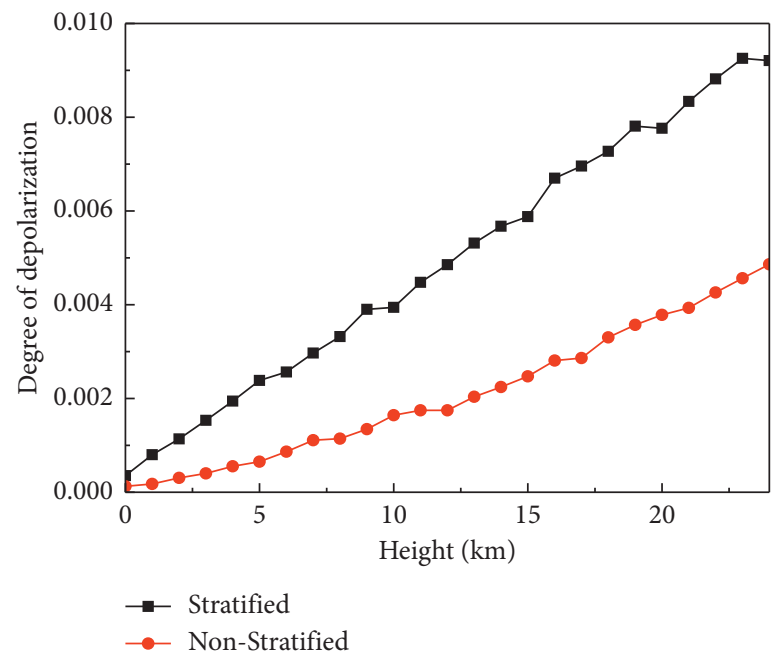

(b)

FIGURE 7: Variation of polarization parameters (stratified and nonstratified) with height $(b=0.1 \mu \mathrm{m})$.

phenomenon of polarized laser is more obvious in the stratified Martian dust environment.

Secondly, the variation of polarization parameter with height is calculated and analyzed, and the horizontally polarized laser at the wavelength of $0.55 \mu \mathrm{m}$ is taken as an example and compared the calculation results in two cases (stratified and nonstratified). The calculation results are shown in Figures 7-9. 


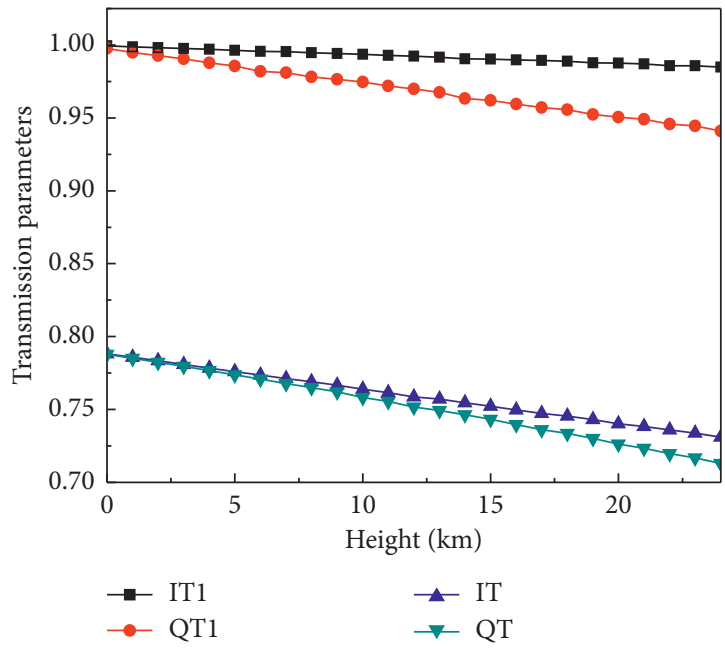

(a)

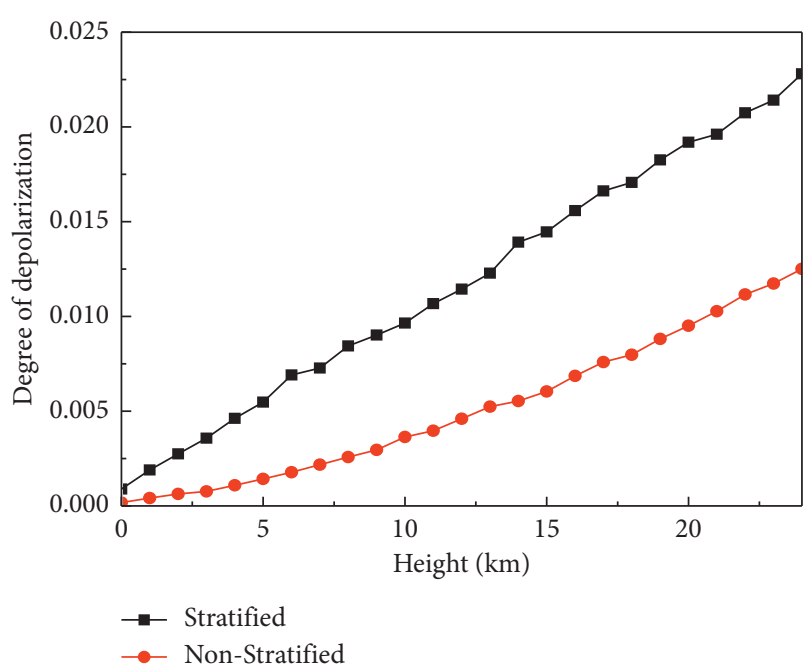

(b)

Figure 8: Variation of polarization parameters (stratified and nonstratified) with height $(b=0.25 \mu \mathrm{m})$.

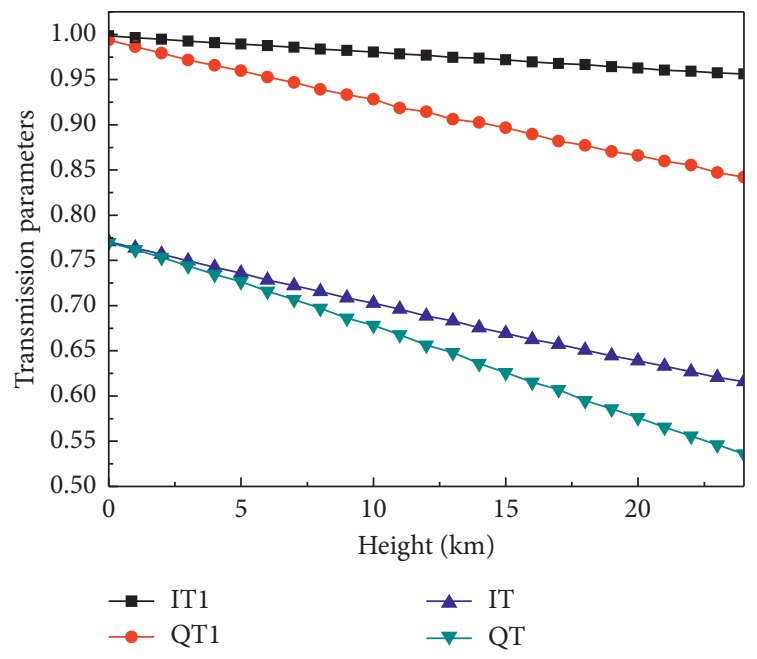

(a)

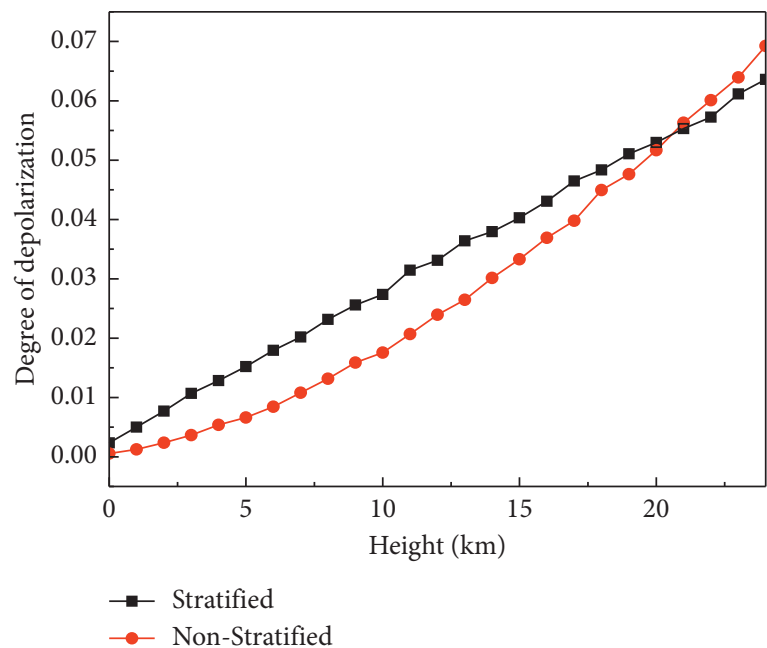

(b)

Figure 9: Variation of polarization parameters (stratified and nonstratified) with height $(b=0.4 \mu \mathrm{m})$.

Figures $7(a), 8(a)$, and $9(a)$ are the variation curves of the transmission parameters with the height of the Martian atmosphere. In these figures, IT1 and QT1 represent the transmission parameters under the condition of stratified, and IT and QT represent the transmission parameters under the condition of nonstratified. It can be seen from Figure 7(a) that the transmission parameter is monotonically decreasing with the increase of height; it also can be seen that the degree of depolarization is monotonically increasing as the increase of height in Figures 7(b) and 9(b), and the degree of depolarization under the condition of stratified is obviously greater than that of nonstratified. On the whole, for different effective variance $b$, the variation trend of the polarization parameters with height for different lase is basically identical. And the transmittance and depolarization degree of polarized laser under the condition of stratified when it transmitted in the Martian dust on Mars are obviously greater than those of nonstratified, which indicated that the polarization parameters calculated under the condition of stratified more accurately reflect the transmission characteristics of polarized laser in the Martian dust environment.

\section{Conclusion}

Based on the vertical distribution characteristic of Martian dust particles, the polarized Monte Carlo method combined with the number concentration and particle size distribution of each layer of dust was used to study the transmission characteristics of polarized laser in the vertical direction of the Martian atmosphere. The transmission transmittance of several typical polarized laser under the condition of 
stratified and nonstratified are calculated, and the variation regulation of horizontal and vertical components of horizontally and vertically polarized laser with transmission distance is given when they are transmitted in Martian dust. When the wavelength is $0.55 \mu \mathrm{m}$, for different effective variance $b$, the depolarization degree under two conditions (stratified and nonstratified) enlarges with the increase of height identically. The depolarization degree of polarized laser under the condition of stratified is significantly greater than that of nonstratified, so it shows that the depolarization phenomenon of polarized laser is more obvious in stratified Martian dust environment. In addition, the real part of the complex refractive index of the wavelength of $7.46 \mu \mathrm{m}$ is the smallest among the six wavelengths, so its transmittance is the biggest when it is transmitted in the Martian dust, while the laser intensity attenuation in the horizontal and vertical components of the wavelength of $0.49 \mu \mathrm{m}$ is the largest, so the trend of intensity attenuation in the vertical and horizontal component is the fastest when it transmitted in the Martian dust.

\section{Data Availability}

Previously reported theoretical and measurement data were used to support this study and are available at [DOI: 10.1364/ opex.13.004420 and DOI: 10.1029/94JE03363]. These prior studies are cited at relevant places within the text as references [1-19].

\section{Conflicts of Interest}

The authors declare that there are no conflicts of interest regarding the publication of this article.

\section{Acknowledgments}

This work was supported by the National Natural Science Foundation of China (61405157) and the Key Industry Innovation Chain Project of Shaanxi Province (2017ZDCXLGY-06-01).

\section{References}

[1] Z. Wu, J. You, and R. Yang, "Study on attenuation characteristics of laser in dust storm," Chinese Journal of Lasers, vol. 31, no. 09, pp. 1075-1080, 2004.

[2] J. Xu and B. Ge, "Simulation and analysis of polarization characteristics of single particle laser scattering," Acta Optica Sinica, vol. 39, no. 04, pp. 413-421, 2019.

[3] M. I. Mishchenko and M. A. Yurkin, "On the concept of random orientation in far-field electromagnetic scattering by nonspherical particles," Optics Letters, vol. 42, no. 3, pp. 494-497, 2017.

[4] G. N. Plass and G. W. Kattawar, "Degree and direction of polarization of multiple scattered light 2: earth's atmosphere with aerosols," Applied Optics, vol. 11, no. 12, pp. 2866-2879, 1972.

[5] G. N. Plass, G. W. Kattawar, and F. E. Catchings, "Matrix operator theory of radiative transfer 1: Rayleigh scattering," Applied Optics, vol. 12, no. 2, pp. 314-329, 1973.
[6] P. Yang, Research on circular polarization modulation technology in atmospheric laser communication, Ph.D. thesis, University of Chinese Academy of Sciences, Changchun, China, 2012.

[7] J. E. Fernández, M. Bastiano, and A. Tartari, "Vector Monte Carlo for simulation of polarized photons," X-Ray Spectrometry, vol. 27, no. 5, pp. 325-331, 2015.

[8] B. Ma, X. Ke, and Y. Zhang, "Research on polarization control and algorithm of beam in coherent optical communication system," Chinese Journal of Lasers, vol. 46, no. 1, pp. 1-8, Article ID 106002, 2019.

[9] Y. Yang, A. Han, and S. Lei, "Simulation of polarized laser transmission characteristics in Martian dust environment," Acta Optica Sinica, vol. 40, no. 13, pp. 197-203, 2020.

[10] G. Alemanno, E. Garcia-Caurel, J. Carter et al., "Determination of optical constants from Martian analog material using a spectro-polarimetric technique," Planetary and Space Science, vol. 195, 2021.

[11] A. Spiga, J. Faure, J.-B. Madeleine, A. Määttänen, and F. Forget, "Rocket dust storms and detached dust layers in the Martian atmosphere," Journal of Geophysical Research: Planets, vol. 118, no. 4, pp. 746-767, 2013.

[12] E. Chassefière, P. Drossart, and O. Korablev, "Post-phobos model for the altitude and size distribution of dust in the low Martian atmosphere," Journal of Geophysical Research Planets, vol. 100, no. E3, pp. 5525-5539, 1995.

[13] R. Acosta-Herazo, J. Monterroza-Romero, M. Á. Mueses, F. Machuca-Martínez, and G. Li Puma, "Coupling the Six Flux Absorption-Scattering Model to the Henyey-Greenstein scattering phase function: evaluation and optimization of radiation absorption in solar heterogeneous photoreactors," Chemical Engineering Journal, vol. 302, pp. 86-96, 2016.

[14] J. C. Ramella-Roman, S. A. Prahl, and S. L. Jacques, "Three Monte Carlo programs of polarized light transport into scattering media: part I," Optics Express, vol. 13, no. 12, p. 4420, 2005.

[15] L. Yang, X. Ke, and D. Ma, "Study on the depolarization of polarized laser in atmospheric propagation," Photoelectric Engineering, vol. 35, no. 11, pp. 62-67, 2008.

[16] L. Wang, Z. Xu, and H. Feng, "Monte Carlo simulation of back-diffusion scattering of polarized laser in polydisperse high concentration medium," Journal of Physics, vol. 54, no. 06, pp. 2694-2698, 2005.

[17] L. Ting, Study on Optical Scattering Characteristics of Atmospheric Suspended Particulate Matter, Xi'an University of Technology, Xi'an, China, 2018.

[18] J. Qin, Study on Laser Scattering Characteristics of Dust Particles in Martian Environment, Xi'an University of Technology, Xi'an, China, 2019.

[19] Y. Yufeng, H. Anli, and C. kangkang, "The transmission characteristic of polarized laser in sandstorm on Mars," in Proceedings of the 2020 IEEE MTT-S International Conference on Numerical Electromagnetic and Multiphysics Modeling and Optimization (NEMO), pp. 1-4, Hangzhou, China, December 2020. 